\title{
Thermodynamics of conformation and deformation of linear polymeric chains in solution
}

\author{
Yu.G.Medvedevskikh \\ The Department of the L.V. Pisarzhevsky Institute of Physical Chemistry \\ of the National Academy of Sciences of Ukraine, \\ 3a Naukova Str., 79053 Lviv, Ukraine
}

Received December 20, 2000

Thermodynamics of conformation and deformation of linear polymeric chains in a solution is built based on the statistics of self-avoiding walks. The entropy and free energy of conformation of a polymeric chain is presented as a sum of two terms. The first one takes into account the contribution of random walk and the second one takes into account the contribution of two limitations, which covered random walk and create the effect of self-organization of a polymeric chain. Deformation of the polymeric chain is considered as an equilibrious transition of Flory ball into conformational ellipsoid. The expressions for thermodynamic and elastic properties of the polymeric chain as functions of the degree of its deformation are suggested. Volumetric module, Young's module and module of polymeric chain shift are expressed through the pressure of conformation; Poisson's ratio depends only upon the dimension of Euclidean space. Forces and work of deformation are determined; the method of calculating the main tensions is suggested.

Key words: conformation, thermodynamics, polymeric chains, deformation modules of elasticity, forces, work

PACS: $05.70 . \mathrm{Ce}$

\section{Introduction}

In many aspects conformation of polymeric chains determines their dynamic and thermodynamic properties in solution. However thermodynamics of deformation of polymeric chains developed in works [1-7] mainly directed towards the analysis and explanation of the elastic properties of caoutchouc has two substantial drawbacks that make it incomplete and not rigorous. Firstly, since it is based on the statistics of random walk (RW) of the end of the of Kuhn chain [4], the effect of exclusive volume, which requires statistics of self-avoiding walk SAW [8] is not considered. Secondly, using an obviously correct statement that the exterior hydrostatic pressure 
cannot considerably influence the conformational condition of a polymeric chain in a solution, this thermodynamics did not take into account the main parameter in the thermodynamic relations, i.e., the volume of the conformation.

Statistics of SAW [8] describes the conformation of an ideal polymeric chain in an ideal solution. According to the statistics, SAW limitation for self-avoidance of trajectories of RW causes such self-organization of polymeric chain when its conformation has got the structure of spherical or, in a more general case, ellipsoid shell, that is Flory radius thick and it is diffusively smeared outside as well as inside the polymeric blob, the core of which is statistically empty for half of Flory radius.

Conformation of ellipsoid can be considered as a deformed condition of Flory ball that permits statistics of SAW to describe thermodynamics of conformation and deformation of a polymeric chain in a solution from general and rigorous point of view.

\section{Entropy and free energy of conformation}

Statistics of SAW on $d$-dimensional lattice is determined by probability $w(s)$ that, by its last step, the trajectory of SAW will end in one of $2^{d}$ equiprobable cells $M_{p}(s)$ which have only different signs of a component vector $s=\left(s_{i}\right), i=1, d$, expression [8]

$$
w(s)=\beta \exp \left\{-N^{2} / d^{d / 2} \prod_{i}\left|s_{i}\right|-\frac{1}{2} \sum_{i} \frac{s_{i}^{2}}{n_{i}}\right\},
$$

in which $\beta^{-1}=e(2 \pi)^{d / 2}$ is the numeral constant, $N$ is the number of sections or length of chain, $d$ is the dimension of Euclidean space, $n_{i}=n_{i}^{+}+n_{i}^{-}$is the number of steps in $i-m$ direction of space including positive $n_{i}^{+}$and negative $n_{i}^{-}, s_{i}=n_{i}^{+}-n_{i}^{-}$ is the number of effective steps.

Numbers $n_{i}, i=1, d$ of steps in all d directions of space and length of chain $N$ are connected by the following relation

$$
\sum_{i} n_{i}=N
$$

Probability $w(s)$ is numerically equal to the shares of those trajectories of SAW from common number $(2 d)^{N}$ of trajectories RW that are possible on $d$-dimensional lattice, which realize the state $M_{p}(s)$. The number of such trajectories determines thermodynamic probability $W(s)$ of realization of the state $M_{p}(s)$ :

$$
W(s)=(2 d)^{N} w(s) .
$$

We introduce a variable of metric shift of the end of chain $x_{i}, i=1, d$ as semi-axis of conformational ellipsoid to whose surface belong the states $M_{p}(s)$ [8]:

$$
x_{i}=a\left|s_{i}\right| d^{1 / 2}
$$


Here $a$ is a statistical length of a section of chain.

Let us denote by $\sigma_{i}$ the standard deviations of Guassian part of distribution (1):

$$
\sigma_{i}^{2}=a^{2} n_{i} d
$$

According to (2) the value of $\sigma_{i}$; is represented by the following relation

$$
\sum_{i} \sigma_{i}^{2}=a^{2} N d
$$

Since $s_{i}^{2} / n_{i}=x_{i}^{2} / \sigma_{i}^{2}, d^{d / 2} \prod_{i}\left|s_{i}\right|=\prod_{i} x_{i} / a^{d}$, distribution (1) can be written in the following form

$$
w(x)=\beta \exp \left\{\frac{-a^{d} N^{2}}{\prod_{i} x_{i}}-\frac{1}{2} \sum_{i} \frac{x_{i}^{2}}{\sigma_{i}^{2}}\right\} .
$$

In continueous $d$-dimensional space, general number $L$ of trajectories of random walk at a given $N$ cannot be determined so simply as in a lattice discrete space. That is why in continueous space the expression for thermodynamic probability $W(x)$ of realization of the state, in which the end of chain is on the surface of conformational ellipsoid with semi-axis $x_{i}$, should be written in the following way

$$
W(x)=L w(x)
$$

Here $L$ is a function only of the length of the chain $N$ and Euclidean space dimension $d$. If we assume that transitions of the end of the chain in the volume $a^{d}$ do not create new states we shall receive in the lattice approximation $L=(2 d)^{N}$. In general, we can assume that order $L$ is determined by the quantity $(2 d)^{N}$.

According to (8) and (7), entropy $S(x)=k \ln W(x)$ of this conformational state can be presented in the following way

$$
\begin{aligned}
S(x) & =S_{0}+S_{\mathrm{s}}(x) \\
S_{0} & =k \ln \beta L \\
S_{\mathrm{s}}(x) & =-k\left\{\frac{a^{d} N^{2}}{\prod_{i} x_{i}}+\frac{1}{2} \sum_{i} \frac{x_{i}^{2}}{\sigma_{i}^{2}}\right\} .
\end{aligned}
$$

The first term $S_{0}$ in (9) is positive and presents entropy of random walk limited only by dimension $\mathbf{d}$ of space and length of chain $N$. The second term $S_{\mathrm{s}}(x)$ in $(9)$ makes negative contribution into entropy of conformation, and the essence of this is that it presents limitations that cover trajectories of random walk. One of these limitations is that self-intersection of trajectories of RW is absent, the second one is that the trajectory must end on the surface of ellipsoid with semi-axis $x_{i}, i=1, d$. These are the limitations which create the effect of self-organization of polymeric chain. That is why $S_{\mathrm{s}}(x)<0$ can be called entropy of self-organization. Thus, conformation of polymeric chain is a statistic form of its self-organization. 
Since statistics of SAW does not take into account the effects of short-range order, the assumption that the free energy of conformation is equal to $F(x)=-T S(x)$ is natural. Therefore

$$
\begin{aligned}
F(x) & =F_{0}+F_{\mathrm{s}}(x) \\
F_{0} & =-k T \ln \beta L \\
F_{\mathrm{s}}(x) & =k T\left\{\frac{a^{d} N^{2}}{\prod_{i} x_{i}}+\frac{1}{2} \sum_{i} \frac{x_{i}^{2}}{\sigma_{i}^{2}}\right\} .
\end{aligned}
$$

For $\beta \ll L$, the expression $F_{0} \approx-k T N \ln 2 d$ is the free energy of the random walk. Taking into account the error of "grating" determination of $L$, the fact that $\ln 2 d \sim d / 2$ and the physical meaning of the quantity $F_{0}$, we assume

$$
F_{0}=-\frac{d}{2} k T N
$$

Thus free energy of the random walk is determined by their progressive energy.

Taking into consideration (15) and (14), we shall rewrite (12) in the following way

$$
F(x)=-\frac{d}{2} k T N+k T\left\{\frac{a^{d} N^{2}}{\prod_{i} x_{i}}+\frac{1}{2} \sum_{i} \frac{x_{i}^{2}}{\sigma_{i}^{2}}\right\} .
$$

Among all possible conformational states we shall further consider the most probable, i.e. the equilibrium ones, which are characterized by the maximum of the entropy and the minimum of the free energy. Minimizing $F(x)$ under the condition $\partial F(x) / \partial x_{i}=\partial F_{\mathrm{s}}(x) / \partial x_{i}=0$, for the equilibrium state we receive the following

$$
-a^{d} N^{2} / x_{i} \prod_{i} x_{i}+x_{i} / \sigma_{i}^{2}=0, \quad i=1, d .
$$

By solving the system of algebraic equations (17), we find equilibrium values of semi-axis of conformational ellipsoid

$$
x_{i}=\sigma_{i}\left(\frac{a^{d} N^{2}}{\prod_{i} \sigma_{i}}\right)^{\alpha}
$$

where

$$
\alpha=1 /(d+2) .
$$

We shall particularly underline the states at which all directions of the random walk of the end of the chain are equilibrium, that is

$$
n_{i}=N / d, \quad i=1, d
$$

Then

$$
\sigma_{i}^{2}=\sigma_{0}^{2}=a^{2} N, \quad i=1, d
$$


Substitution of (21) into (18) makes semi-axis $x_{i}$ of the equilibrium ellipsoid equal to the radius of Flory, $x_{i}=R_{\mathrm{f}}, i=1, d$,

$$
R_{\mathrm{f}}=a N^{\nu}
$$

with Flory index $\nu=3 \alpha$ or

$$
\nu=\frac{3}{(d+2)}
$$

The expression of Flory (22) is a particular case of (18) and is true only at equal probability of the random walk in all directions of $d$-dimensional space. If this condition (20) is not kept equilibrium conformation of polymeric chain acquires the form of ellipsoid with semi-axes $x_{i}$, determined by (18). Therefore as a measure of deformation of polymeric chain that characterizes deviation of its conformational state from Flory ball it is convenient to take the following variables

$$
\begin{aligned}
\psi_{i} & =\sigma_{i} / \sigma_{0}, \\
u & =\prod_{i} \psi_{i} .
\end{aligned}
$$

From (6) and (21) it follows that value $\psi_{i}$ is covered by the following relation

$$
\sum_{i} \psi_{i}^{2}=d
$$

According to (26) the function $u=\prod_{i} \psi_{i}$ has the maximal value of $u=1$ for Flory ball, when all $\psi_{i}=1$; at all the other legitimate values of $\psi_{i}$ according to (26) the function $u<1$. Consequently any deformation of Flory ball is accompanied by decrease of $u=\prod_{i} \psi_{i}$.

Using (18), we shall express semi-axes $x_{i}$ of equilibrious ellipsoid through parameters $\psi_{i}$ and $u$ :

$$
x_{i}=R_{\mathrm{f}} \psi_{i} / u^{\alpha} .
$$

Having introduced multiplicity of linear deformation in $i$ direction

$$
\lambda_{i}=\frac{x_{i}}{R_{\mathrm{f}}}
$$

we shall receive

$$
\prod_{i} \lambda_{i}=u^{2 \alpha}
$$

The equation of the relation $\lambda_{i}$ corresponds to the equation of the relation (26) between $\lambda_{i}$ :

$$
\sum_{i} \lambda_{i}^{2}=d / \prod_{i} \lambda_{i}
$$

We shall receive the expression for free energy $F$ of equilibrium conformation of polymeric chain by substituting (27) into (16):

$$
F=-\frac{d}{2} k T N+\left(1+\frac{d}{2}\right) \cdot k T\left(\frac{R_{\mathrm{f}}}{\sigma_{0}}\right)^{2} / u^{2 \alpha} .
$$


For Flory ball we have

$$
F_{\mathrm{f}}=-\frac{d}{2} k T N+\left(1+\frac{d}{2}\right) \cdot k T\left(\frac{R_{\mathrm{f}}}{\sigma_{0}}\right)^{2} .
$$

Here

$$
\left(R_{\mathrm{f}} / \sigma_{0}\right)^{2}=N^{(4-d) /(d+2)} .
$$

As one can see, with the increase of the Flory ball deformation free energy of equilibrium conformation $F$ increases and it happens at the expense of the free energy of self-organization $F_{\mathrm{s}}$ :

$$
F_{\mathrm{s}}=(1+d / 2) k T\left(R_{\mathrm{f}} / \sigma_{0}\right)^{2} / u^{2 \alpha} .
$$

For the Flory ball

$$
F_{\text {sf }}=(1+d / 2) k T\left(R_{\mathrm{f}} / \sigma_{0}\right)^{2} .
$$

At a given $d$ and $N$ the change of the free energy at the equilibrium deformation of the Flory ball is determined as a difference $\Delta F=F-F_{\mathrm{f}}=F_{\mathrm{s}}-F_{\mathrm{sf}}$. Therefore according to (34) and (35) we have

$$
\Delta F=(1+d / 2) k T\left(R_{\mathrm{f}} / \sigma_{0}\right)^{2}\left(1 / u^{2 \alpha}-1\right) .
$$

At any deformations of Flory ball $\Delta F>0$, subsequently the work is done with the system.

For $d=3$-dimensional space we have

$$
\Delta F=\frac{5}{6} k T N^{1 / 5}\left(\lambda_{x}^{2}+\lambda_{y}^{2}+\lambda_{z}^{2}-3\right) .
$$

Let us compare (37) with the well-known expression of work $W$ of deformation of caoutchouc volume unit [5]

$$
W=\frac{1}{2} k T N_{\mathrm{c}}\left(\lambda_{x}^{2}+\lambda_{y}^{2}+\lambda_{z}^{2}-3\right) .
$$

where $N_{\mathrm{c}}$ is the number of chain per unit of volume.

Similarity of the expressions (37) and (38) is obvious enough. But substantial differences are more important. The first difference is that in (37) the multiplier $N^{1 / 5}$ is presented. It appears only in RWSA statistics and indicates that as the chain grows longer the work of Flory ball deformation increases. The second difference is that because of the presence of the multiplier $N_{\mathrm{c}}$ the work $W$ has dimensions of pressure, tension, etc., which are the characteristics of elastic properties of caoutchouc. That is why (38) is also called to be the expression of highly elastic potential of rubber $[6,7]$. We shall see later that the elastic properties of polymeric chains are described by equations different from (38) by their form. Finally we shall note that in the interpretation of (38) the condition of incompressibility of caoutchouc in the form of $\lambda_{x} \lambda_{y} \lambda_{z}=1$ is used. According to the relation (30), in such a case the expression in brackets (38) should be equal to 0, i.e., without the change of configuration volume there is no deformation work. 


\section{The equation of state of equilibrium conformation}

Leaving out numeral coefficients, we shall determine the volume $V_{\mathrm{f}}$ of Flory ball in $d$-dimensional space using of the following expression

$$
V_{\mathrm{f}}=R_{\mathrm{f}}^{d},
$$

and the volume $V$ of conformational ellipsoid with the expression

$$
V=\prod_{i} x_{i}
$$

Thus, taking into account (27) we shall receive

$$
V=V_{\mathrm{f}} u^{2 \alpha}=R_{\mathrm{f}}^{d} u^{2 \alpha} .
$$

So, at any deformations of Flory ball, the volume of configuration decreases.

Having introduced multiplicity of volumetric deformation by the relation $\lambda_{V}=$ $V / V_{\mathrm{f}}$, we have

$$
\lambda_{v}=u^{2 \alpha}=\prod_{i} \lambda_{i}
$$

Outer hydrostatic pressure cannot considerably influence the conformational state of a polymeric chain in the solution. But at deformation of Flory ball, the volume of the conformation decreases and the free energy increases. Subsequently, according to thermodynamic relation $\partial F / \partial V=-P$ there should exist the measure of connection between free energy $F$ and volume $V$ of configuration, which plays the role of conformation pressure. Thus pressure $P$ of conformation is determined by the following expression

$$
\partial F / \partial V=\partial F_{\mathrm{s}} / \partial V=-P .
$$

Differentiating (31) or (34) and taking into account (41), we receive

$$
P=(1+d / 2) k T\left(R_{\mathrm{f}} / \sigma_{0}\right)^{2} / R_{\mathrm{f}}^{d} u^{4 \alpha}
$$

For Flory ball

$$
P_{\mathrm{f}}=(1+d / 2) k T\left(R_{\mathrm{f}} / \sigma_{0}\right)^{2} / R_{\mathrm{f}}^{d} .
$$

Comparing (41) and (44), we shall receive the equation of conformational state of polymeric chain in the solution

$$
P V^{2}=\text { const }
$$

where

$$
\text { const }=\left(1+\frac{d}{2}\right) k T\left(R_{\mathrm{f}} / \sigma_{0}\right)^{2} R_{\mathrm{f}}^{d}
$$

On the other hand, comparing (41) and (44) with the expression for $F_{\mathrm{s}}(34)$, we find

$$
F_{\mathrm{s}}=P V
$$


and subsequently pressure of conformation $P=F_{\mathrm{s}} / V$ is numerically equal to the density of free energy of self-organization of polymeric chain.

From (48) taking into account (46) and (47) we have

$$
F_{\mathrm{s}} V=\text { const. }
$$

Thus, values $P V^{2}$ and $F_{\mathrm{s}} V$ are integrals of the process of equilibrious deformation of polymeric chain.

\section{Modules of elasticity of polymeric chain}

Within the approximation of isotropy of polymeric chain, its relative deformation $\partial x_{i} / x$ in $i$ direction of $d$-dimensional space under the influence of all main forces $f_{i}$, $i=1, d$ in differential form is described by the expression [9]

$$
Y \partial x_{i} / x_{i}=\partial f_{i} / \prod_{j \neq i} x_{j}+\gamma \sum_{j \neq i} \partial f_{j} / \prod_{k \neq j} x_{k}
$$

where $Y$ is Young's module, $\gamma$ is Poisson's ratio, $\prod_{j \neq 1} x_{j}$, and $\prod_{k \neq j} x_{k}$ are the values of the areas in $d$-dimensional space that are normal to the forces $f_{i}$ and $f_{j}$ correspondingly.

Let us rewrite (50) with regard to Young's module in the form

$$
Y=\frac{x_{i}^{2}}{\prod_{i} x_{i}} \frac{\partial f_{i}}{\partial x_{i}}+\gamma \sum_{j \neq i} \frac{x_{i} x_{j}}{\prod_{i} x_{i}} \frac{\partial f_{j}}{\partial x_{i}} .
$$

Equations (50) and (51) are written in the system of signs of mechanics according to which $f_{i}=\partial F(x) / \partial x_{i}$. Differentiating (16), we have

$$
f_{i} / k T=-a^{d} N^{2} / x_{i} \prod_{i} x_{i}+x_{i} / \sigma_{i}^{2}
$$

At equilibrium deformation in any current conformational state, the forces should be equal to 0 , and this is expressed by the condition of equilibrium (17). But derivatives $\partial f_{i} / \partial x_{i}$ and $\partial f_{j} / \partial x_{i}$ as factors of equilibrium process are not equal to zero. That is why differentiating (52) with respect to $x_{j}$ and $x_{j}$ and substituting into the resulting expressions equilibrious values $x_{i}, i=1, d$ by (18) and (27), we have

$$
\begin{aligned}
\partial f_{i} / \partial x_{i} & =3 k T / \sigma_{0}^{2} \psi_{i}^{2} \\
\partial f_{j} / \partial x_{i}=\partial f_{i} / \partial x_{j} & =k T / \sigma_{0}^{2} \psi_{i} \psi_{j} .
\end{aligned}
$$

Substitution of (53) and (53) into (51) gives

$$
Y=k T(3+\gamma(d-1))\left(R_{\mathrm{f}} / \sigma_{0}\right)^{2} / R_{\mathrm{f}}^{d} u^{4 \alpha} .
$$

For Flory blob, Young's module is equal to

$$
Y_{\mathrm{f}}=k T(3+\gamma(d-1))\left(R_{\mathrm{f}} / \sigma_{0}\right)^{2} / R_{\mathrm{f}}^{d},
$$


that is why (55) can be written in the following form

$$
Y=Y_{\mathrm{f}} / u^{4 \alpha}=Y_{\mathrm{f}} / \lambda_{v}^{2}
$$

As one can see from comparison of (44) and (53), Young's module and pressure are different only by numerical constants. In case of $d=3$-dimensional space, the connection between Young's module and pressure is expressed by volume module $E=-V \partial P / \partial V$ using the correlation $E=Y / 3(1-2 \gamma)$. Taking into account the logics of its derivation [9], for $d$-dimensional space we can write

$$
E=Y / d(1-\gamma(d-1))
$$

From the equation of state (46), it follows

$$
E=-V \partial P / \partial V=2 P .
$$

Thus,

$$
Y=2(3+\gamma(d-1)) P /(d+2) .
$$

Using expressions (45) and (58), we shall solve (63) with regard to $\gamma$

$$
\gamma=(d+3) /(d+1)^{2} .
$$

As one can see, Poisson's ratio $\gamma$ depends only upon dimension $d$ of Euclidean space. Namely, for $d=3,2$ and 1 we have $\gamma=3 / 8,5 / 9$ and 1 ; accordingly $Y=3 / 2 P$, $16 / 9 P$ and $2 P$. As it was expected, in $d=1$-dimensional space, Young's module coincides with volume module. Through Young's module and Poisson's ratio we find module of shift $\mu(d \geqslant 2)[9]$

$$
\mu=Y / 2(1+\gamma)
$$

For $d=3$-dimensional space $\mu=4 / 11, Y=6 / 11 P$.

\section{Young's module of caoutchouc}

It would be incorrect to make direct theoretic continuation of elastic properties of polymeric chain in a solution in order to describe elastic properties of caoutchouc. But we can suggest a semi-empirical approach as in $[4,5]$. Thus, in the expression (55) of Young's module of polymeric chain in a solution instead of blob volume $R_{\mathrm{f}}^{d}$, that is theoretically calculated using Flory formula (22), empirical volume of polymeric chain in caoutchouc is introduced

$$
1 / R_{\mathrm{f}}^{d}=\rho / M N_{\mathrm{A}},
$$

where $\rho$ is the density of caoutchouc; $M$ is the molecular mass of polymeric chain; $N_{\mathrm{A}}$ is Avogadro number.

With such a substitution, the expression for Young's module of caoutchouc in $d=3$-dimensional space $(\gamma=3 / 8)$ acquires the following semiempirical form

$$
Y_{\mathrm{c}}=3.75 R T N^{1 / 5}\left(\frac{\rho}{M}\right) / u^{4 \alpha} .
$$




\section{Main tensions}

The relation between tension $G_{i}$ in the plane normal to $i$ direction of deformation and its relative value $\partial x_{i} / x_{i}$ in differential form is described by the following expression

$$
\partial G_{i}=Y \partial x_{i} / x_{i}
$$

The analysis shows that (65) has no analytical solution in general form, but it permits to get the necessary equations of connection. We shall receive the first equation of connection by substituting Young's module in the form of (57) and taking the sum of (65) in all directions of $d$-dimensional space:

$$
\sum_{i} G_{i}=-\left(Y_{\mathrm{f}} / 2\right)\left(1 / u^{4 \alpha}-1\right) .
$$

Thus, the sum of main tensions is negative, which is the result of the decrease of conformational volume of polymeric chain. Taking into account the relation (29), equation (66) can acquire the following form

$$
\sum_{i} G_{i}=-\left(Y_{\mathrm{f}} / 2\right)\left(1 / \prod_{i} \lambda_{i}^{2}-1\right) .
$$

The second equation of relation between $G_{i}$ we shall find as follows

$$
\prod_{i} G_{i}=(-1)^{d}\left(Y_{\mathrm{f}} / 2 d\right)^{d} \prod_{i}\left(1 / \lambda_{i}^{2 d}-1\right) .
$$

At additional suggestions concerning the character of deformation of polymeric chain simultaneous solution of (67) and (68) enables us to calculate all main tensions $G_{i}$.

\section{Forces and work of deformation}

We shall define the force of deformation as the force that should be applied to Flory blob, the radius of which $R_{\mathrm{f}}$ is equilibrium with respect to the values $\sigma_{i}=\sigma_{0}, i=1, d$, in order to transform it into the state of ellipsoid with semi-axes $x_{i}$, equilibrium with regard to values $\sigma_{i} \neq \sigma_{0}, i=1, d$. According to this definition in expression (53) one should use $\sigma_{i}=\sigma_{0}, i=1, d$, with the values of $x_{i}$ taken according to (18) as equilibrium with respect to $\sigma_{i} \neq \sigma_{0}$. Making necessary substitution in (53), we shall get the expression for force of deformation

$$
f_{i}=k T\left(R_{\mathrm{f}} / \sigma_{0}^{2}\right)\left(\psi_{i}^{2}-1\right) / \psi_{i} u^{\alpha} .
$$

According to the adopted system of signs $f_{i}>0$ at stretch $\left(\psi_{i}>1\right)$ and $f_{i}<0$ at compression $\left(\psi_{i}<1\right)$.

Forces of deformation are not arbitrary, they satisfy the following relations

$$
\sum_{i} f_{i} \psi_{i}=0, \quad \sum_{i} f_{i} \lambda_{i}=0 .
$$


Work of deformation in all main directions is determined by the expression

$$
A=\sum_{i} \int_{R_{\mathrm{f}}}^{x_{i}} f_{i} \partial x_{i}
$$

Substitution of (69) into (71) and taking into account (27) gives

$$
A=k T\left(R_{\mathrm{f}} / \sigma_{0}\right)^{2} \int_{R_{\mathrm{f}}}^{x_{i}}\left(\sum_{i} \frac{x_{i} \partial x_{i}}{R_{\mathrm{f}}^{2}}-\sum_{i} \frac{\partial x_{i}}{x_{i} u^{2 \alpha}}\right) .
$$

Having integrated (72) we shall receive

$$
A=(1+d / 2) k T\left(R_{\mathrm{f}} / \sigma_{0}\right)^{2}\left(1 / u^{2 \alpha}-1\right),
$$

that is identical to (36): $A=\Delta F$. This relation proves the correctness of determining the deformation force in (69).

\section{Calculations and illustrations}

Let us limit ourselves only to calculations of thermodynamic and elastic properties of polymeric chain in real $d=3$-dimensional Euclidean space. But in this case let us consider a particular situation, though obviously the most typical one.

Let us assume that among three main forces $f_{x}, f_{y}$ and $f_{z}$ only one, let it be $f_{z}$, is an independent variable, i.e. the outside force that influences the polymeric chain. Then forces $f_{x}$ and $f_{y}$ will be reactions for $f_{z}$. If $f_{z}>0$ and stretch of polymeric chain takes place along the axis $z$, then reactions $f_{x}$ and $f_{y}$ are negative and along the axes $x$ and $y$ polymeric chain compresses. If polymeric chain is isotropic, then forces and multiplicities of linear deformations along the axes $x$ and $y$ will be equal: $f_{x}=f_{y}, \lambda_{x}=\lambda_{y}$. Conformational state of such a polymeric chain has the form of a prolonged (along the axis $z$ ) ellipsoid of rotation. And, on the contrary, if $f_{z}<0$ and polymeric chain compresses along the axis $z$, then $f_{x}=f_{y}>0$ and polymeric chain stretches along the axes $x$ and $y$ transferring according to the condition $\lambda_{z}<1$, $\lambda_{x}=\lambda_{y}>1$ into oblate (along the axis $z$ ) ellipsoid of rotation.

For ellipsoid of rotation not only $\lambda_{x}=\lambda_{y}$, but also $\psi_{x}=\psi_{y}$. That is why the main equations of relations (26) and (30) acquires the following form

$$
\begin{aligned}
2 \psi_{x}^{2}+\psi_{z}^{2} & =3 \\
2 \lambda_{x}^{2}+\lambda_{z}^{2} & =3 / \lambda_{x}^{2} \lambda_{z} .
\end{aligned}
$$

Here $\alpha=1 / 5$,

$$
\begin{aligned}
& u=\psi_{x}^{2} \psi_{z} \\
& \lambda_{x}=\psi_{x} /\left(\psi_{x}^{2} \psi_{z}\right)^{1 / 5}, \quad \lambda_{z}=\psi_{z} /\left(\psi_{x}^{2} \psi_{z}\right)^{1 / 5} \\
& \lambda_{v}=\left(\psi_{x}^{2} \psi_{z}\right)^{2 / 5}=\lambda_{x}^{2} \lambda_{z} .
\end{aligned}
$$




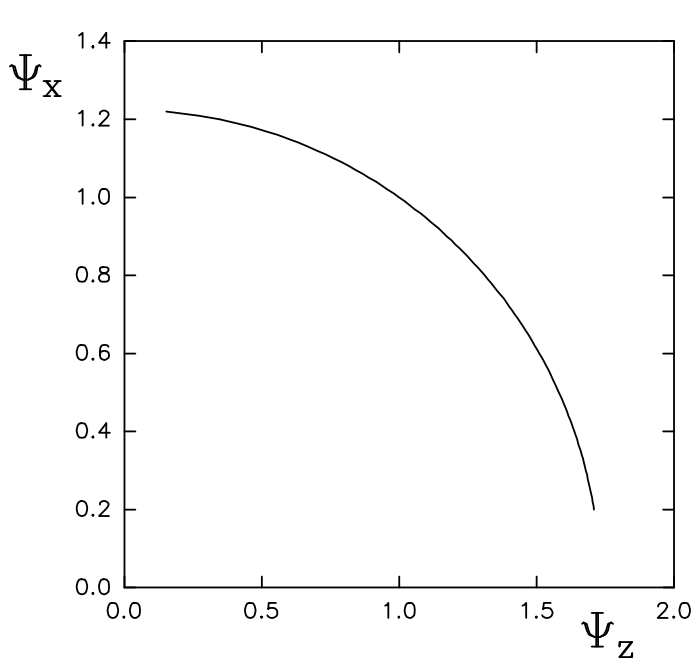

Figure 1. Relationship between parameters $\psi_{x}$ and $\psi_{z}$ of equation (74) during the expansion $\left(\psi_{z}>1\right)$ and pressure $\left(\psi_{z}<1\right)$ of polymer chain at $z$ axis.

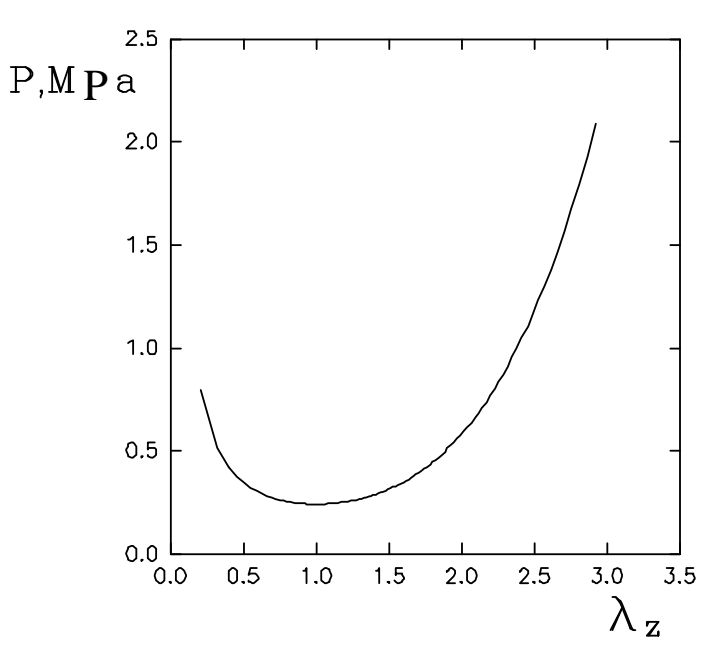

Figure 3. Pressure $P$ of polymer chain conformation during its expansion $\left(\lambda_{z}>1\right)$ and pressure $\left(\lambda_{z}<1\right)$ at $\mathrm{z}$ axis. $N=243, a=0.186 \mathrm{~nm}, T=298 \mathrm{~K}$.

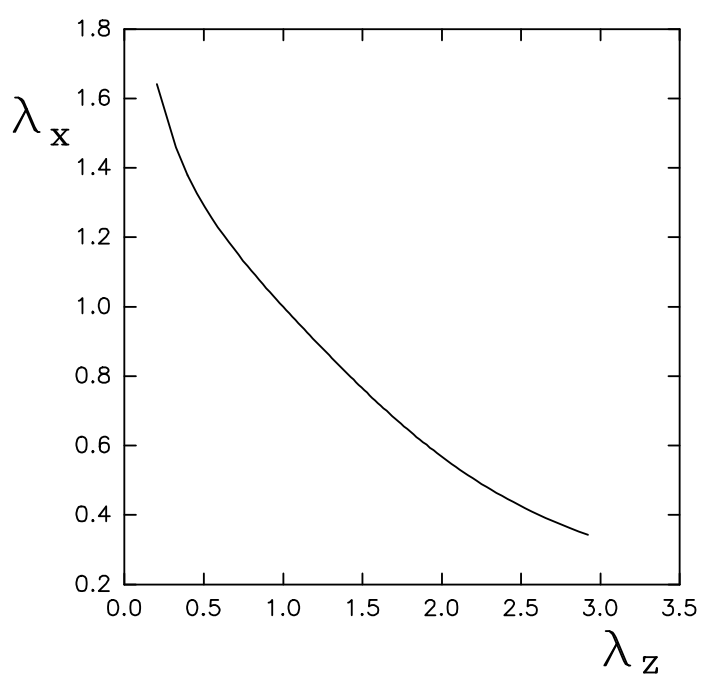

Figure 2. Relationship between degrees of linear deformations $\lambda_{x}$ and $\lambda_{z}$ of equation (75) during the expansion $\left(\lambda_{z}>1\right)$ and pressure $\left(\lambda_{z}<1\right)$ of polymer chain at $z$ axis.

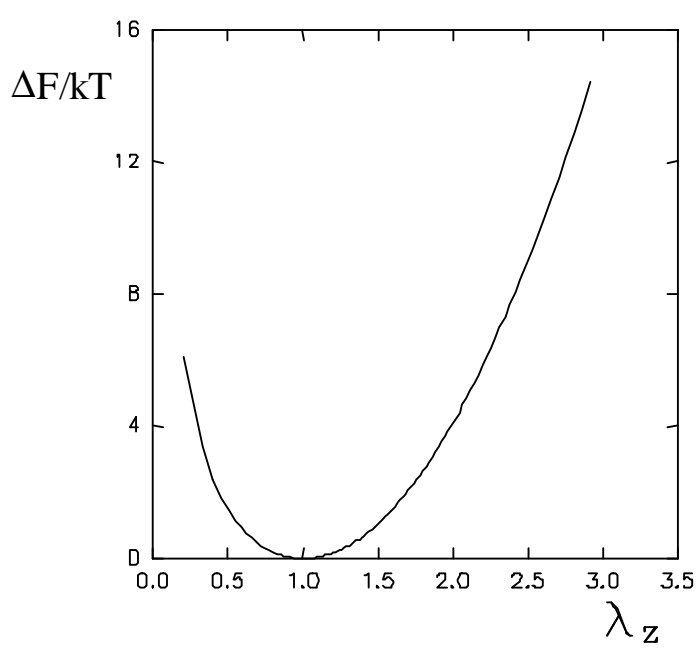

Figure 4. Work $A=\Delta F$ of polymer chain deformation during it expansion $\left(\lambda_{z}>1\right)$ and pressure $\left(\lambda_{z}<1\right)$ at $\mathrm{z}$ axis. $N=243$. 


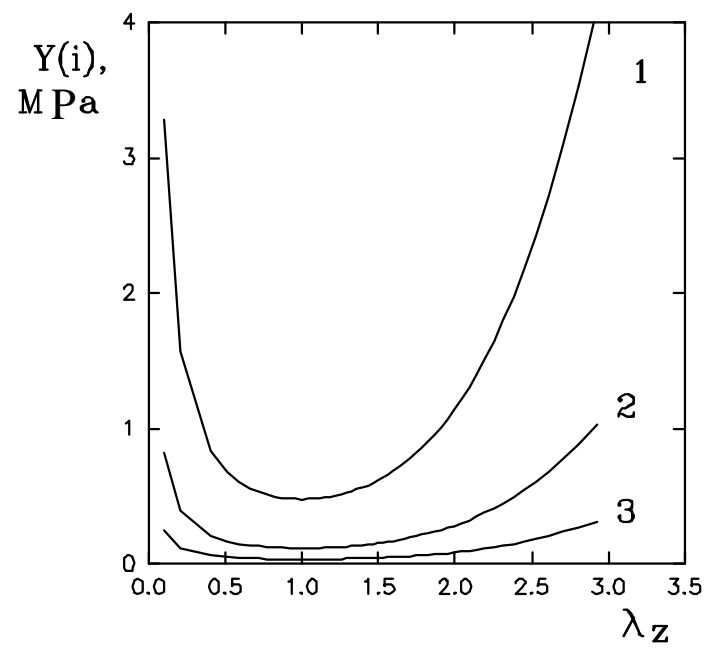

Figure 5. Young's module of polymer chains in solution $(2-a=0.186 \mathrm{~nm}, 3-$ $a=0.125 \mathrm{~nm}$, calculation as $3 P / 2)$ and caoutchouc $(1-$ calculation by equation (64), $a=0.125 \mathrm{~nm}) . N=1024, T=298 \mathrm{~K}$.

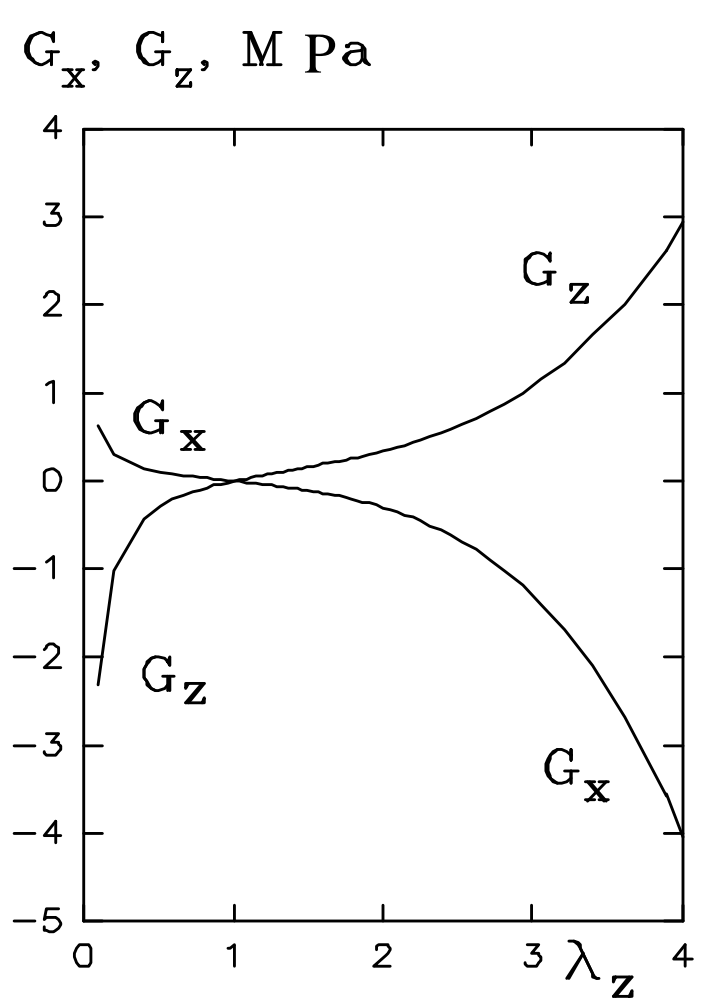

Figure 6. The main exertions $G_{x}$ and $G_{z}$ during the expansion $\left(\lambda_{z}>1\right)$ and pressure $\left(\lambda_{z}<1\right)$ of polymer chain at $z$ axis. $N=243, a=0.186 \mathrm{~nm}, T=$ $298 \mathrm{~K}$.

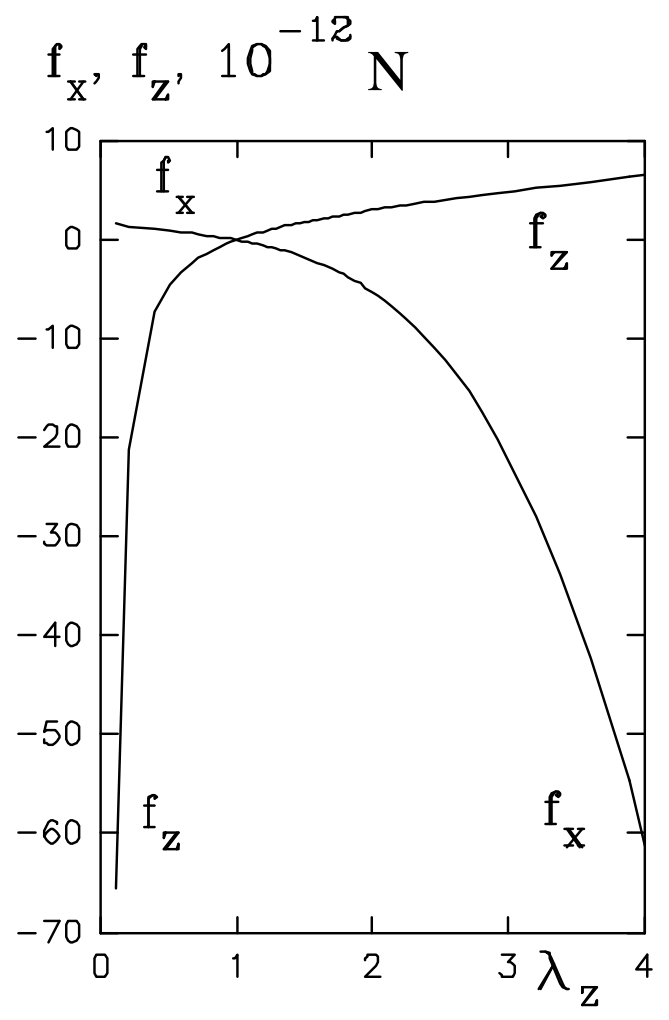

Figure 7. Deformation forces during the expansion $\left(\lambda_{z}>1\right)$ and pressure $\left(\lambda_{z}<1\right)$ of polymer chain at $z$ axis. Calculation by equation (69): $N=243$, $a=0.186 \mathrm{~nm}, T=298 \mathrm{~K}$. 
The relation between parameters $\psi_{x}$ and $\psi_{z}$ is shown in figure 1 , between $\lambda_{x}$ and $\lambda_{z}$ - in figure 2. The expressions (74)-(77) create the basis for calculating all thermodynamic and elastic properties of polymeric chain. In figures 3 and 4 the relationship of pressure of conformation $P=5 / 2 k T / a^{3} N^{8 / 5} \lambda_{\nu}^{2}$ and work of deformation $A=\Delta F=5 / 2 k T N^{1 / 5}\left(1 / \lambda_{\nu}-1\right)$ is presented. The conditions of calculations are indicated in cutlines.

Young's module for polymeric chains in solution is calculated as $3 P / 2$, for caoutchouc - according to semi-empirical equation (64). The results of the work are compared in figure 5 .

The equation of relation of (67) and (68) between main tensions $G_{x}$ and $G_{z}$ acquire the form

$$
\begin{aligned}
2 G_{x}+G_{z} & =-\frac{1}{2} Y_{\mathrm{f}}\left(1 / \lambda_{v}^{2}-1\right) \\
G_{x}^{2} G_{z} & =-\left(Y_{\mathrm{f}} / 6\right)^{3}\left(1 / \lambda_{x}^{6}-1\right)^{2}\left(1 / \lambda_{z}^{6}-1\right) .
\end{aligned}
$$

Simultaneous solution of right parts of (79) and (80) leads to solving of an abridged cubic equation.

The results of calculations $G_{x}$ and $G_{z}$ are presented in figure 6 .

The forces of deformation $f_{x}$ and $f_{y}$ are calculated according to the equation (77) and illustrated in figure 7 . As short comments, we shall note that for three times stretch of polystyrene molecular in the solution $(N=243)$ along the axis $z\left(\lambda_{z}=3\right)$ it is necessary to apply forces of deformation $f_{z} \approx 5 \cdot 10^{-12} \mathrm{H}$, which is equal to gravity force created by mass $5 \cdot 10^{-13} \mathrm{~g}$. Mass of polystyrene molecular is equal to $\sim 4 \cdot 10^{-20} \mathrm{~g}$. So, elastic properties of polymeric chain in the solution are extensive enough.

\section{References}

1. Kuhn W. // 1936, vol. 76, p. 258.

2. James H.M., Guth E. // J. Chem. Phys., 1943, vol. 11, p. 455.

3. Treloar L.R.G. // Trans. Faraday Soc., 1943, vol. 39, p. 36; vol. 39, p. 241.

4. Kuhn W. // Koll. Zs., 1934, vol. 68, p. 2.

5. Treloar L. The Physics of Rubber Elasticity. Oxford, 1949.

6. Bartenev G.M., Frenkel C.Ya. Physics of Polymers. Leningrad, Chimiya, 1990 (in Russian).

7. Askadskiy A.A. Deformation of Polymers. Moscow, Chimiya, 1973 (in Russian).

8. Medvedevskikh Yu.G. // Condens. Matter Phys, 2001, vol. 4, No. 2(26), p. 209.

Statistics of linear polymer chains in the model of random wanderings excluding selfintersection (in Russian).

9. Feynman R., Leighton R., Sands M. The Feynman Lectures of Physics. vol. 2. Massachusetts, 1964. 


\section{Термодинаміка конформації та деформації лінійних полімерних ланцюгів у розчині}

\section{Ю.Г.Медведевських}

Відділення Інституту фізичної хімії

ім. Л.В.Писаржевського НАН України

79053 Львів, вул. Наукова, За

Отримано 20 грудня 2000 р.

Термодинаміка конформації і деформації лінійних полімерних ланцюгів у розчині побудована на основі статистики блукань без перехрещення. Остання виражає ентропію та вільну енергію конформації полімерного ланцюга у вигляді суми доданків, один з яких враховує внесок випадкових блукань, інший - двох обмежень, що накладаються на випадкові блукання і створюють ефект самоорганізації полімерного ланцюга. Його деформація розглянута у вигляді рівноважного переходу клубка Флорі в конформаційний еліпсоїд. Запропоновані вирази термодинамічних та пружних властивостей полімерного ланцюга як функцій ступеня його деформації. Об'ємний модуль, модулі Юнга та зсуву полімерного ланцюга виражаються через тиск конформації, коефіцієнт Пуассона залежить тільки від розмірності евклідового простору. Визначені сили та робота деформації, запропонований метод розрахунку головних напружень.

Ключові слова: конформація, полімерний ланцюг, термодинаміка, деформація, пружні властивості, сила, робота

PACS: 05.70.Ce 
